\title{
Nanoscale
}

\section{Electroosmotic flow in single PDMS nanochannels †}

\author{
Ran Peng ${ }^{\mathrm{a}}$ and Dongqing $\mathrm{Li}^{* \mathrm{a}}$
}

Received 00th January 20xx, Accepted 00th January 20xx

DOI: $10.1039 / \times 0 \times x 00000 x$

www.rsc.org/

\begin{abstract}
Electroosmotic flow (EOF) velocity in single PDMS nanochannels with a dimension as small as $20 \mathrm{~nm}$ is investigated systematically by the current slope method in this paper. A novel method for fabrication of single nanochannels on PDMS surfaces is developed. The effects of channel size, ionic concentration of electrolyte solution and electric field on the EOF velocity in single nanochannels are investigated. The results show that EOF velocity in smaller nanochannels with overlapped electric double layers (EDL) is proportional to the applied electric field but is smaller than the EOF velocity in microchannels under the same applied electric field. EOF velocity in relatively large nanochannels without the overlap of EDLs is independent of the channels size and is the same as that in microchannels under the same applied electric field. Furthermore, in smaller nanochannels with overlapped EDLs, EOF velocity depends on the ionic concentration and also on the channel size. The experimental results reported in this paper are valuable for the future studies of electrokinetic nanofluidics.
\end{abstract}

\section{Introduction}

Electroosmotic flow (EOF) describes the electrokinetically driven liquid motion due to the existence of electric double layer (EDL). EOF in microchannels plays an important role in microfluidics and has been studied comprehensively. In microchannels, the thickness of EDL is generally very small and is negligible in comparison with the channel size in most cases. However, as the dimensions of nanochannels are comparable with the thickness of EDL, EDLs may overlap and consequently many new transport phenomena such as current rectification ${ }^{1-}$ ${ }^{4}$, ion enrichment and depletion ${ }^{5}$, new dynamics of pressure driven flow ${ }^{6-8}$ have been discovered. These new transport phenomena facilitate various novel applications and studies of the nanofluidics 9-12. Theoretically, in nanochannels with overlapped EDLs, traditional Poisson-Boltzmann equation and Helmholtz-Smoluchowski equation used in microchannel systems are not applicable anymore ${ }^{13}$. For the case of extremely small nanochannels, even the continuum hypothesis can hardly survive when the channel size is in the order of the interaction length of the fluid molecules ${ }^{12}$.

Over the past few decades, a series of analytical and numerical models has been developed to study the effects of overlapping of EDLs ${ }^{11,14-20}$, ionic size ${ }^{21}$, ionic valence ${ }^{22,23}$, viscosity 24,25 and $\mathrm{pH}$ value 26,27 of the solutions, surface roughness 28,29 and surface charge density $13,16,30-32$ of the channel walls, and the channel size ${ }^{13,33}$ on the electroosmotic flow in nanochannels. Qu and $\mathrm{Li}^{14}$ derived an overlapped EDL model to calculate electrical potential and ion distribution in the

a. Department of Mechanical and Mechatronics Engineering, University of Waterloo, Waterloo, Ontario, Canada, N2L3G1. Email: dongqing@uwaterloo.ca + Electronic Supplementary information (ESI) available: Simulation of

electroosmotic flow (EOF) in nanochannels. diffuse layer based on the Debye-Huckel approximation. Baldessari ${ }^{19,34}$ also set a new model to predict electric potential distribution in overlapped EDLs with the consideration of ion mobility and $\mathrm{pH}$ of the solution. In recent years, molecular dynamics (MD) method combining with continuum theory has also been used to simulate ion distributions in EDLs and EOF in nanochannels, and found that ion size of the solvent strongly affects the ion distribution near the channel surfaces ${ }^{33}$. Qiao 24 argued that the electroosmotic mobility in nanochannels increases with the surface charge of the channel surfaces, however, the speed is slower than that predicted by the continuum theory due to the increasing viscosity in the interfacial layer. Liu and Sofos 28,29 studied the channel roughness effects upon the electroosmotic flow in nanochannels numerically, showing that even a small roughness value can reduce the electroosmotic flow velocity dramatically and give rise to a non-Newtonian behaviour of the fluid. Pennathur and Santiago ${ }^{23,35}$ studied EOF and ion transport in nanochannels theoretically, proved that when the thickness of the EDL is comparable to the channel size, the electroosmotic flow velocity profile in the nanochannel is nonuniform, and a decrement of EOF velocity occurs when EDLs get overlapped. Bhattacharyya 18 investigated electroosmotic flow in nanochannels in which the channel height and channel width are all on the order of the thickness of EDL and predicted that the Debye-length is not a good parameter to estimate the actual thickness of EDL. Modeling and simulation of ionic currents in 3D nanomembrane connected with microchannels was performed by Chatterjee et al ${ }^{36}$, showing that electrokinetic flow in hybrid micro-nanofluidic system is dependent on the capacitance of the nanomembrane and the surface charge of the nanoarrays. Movahed and $\mathrm{Li}^{13}$ presented a 3-D model on EOF transport phenomena in nanochannels, showing that flow 
field, electrical field and concentration field in nanochannels are all dependent on the channel size.

Experimental results of mass transfer in nanochannels are very limited. Stein 37 explored ion transport in silica nanochannels of $70 \mathrm{~nm}$ in height and $50 \mu \mathrm{m}$ in width. Their work found that the conductance of the nanochannels saturates at dilute limit, and the conductance of the nanochannels is independent of the ion concentration, and ion transport properties in nanochannels is dominated by the surface charge. Ion transport in nanochannels of $2 \mathrm{~nm}$ deep was conducted by Duan and Majumdar ${ }^{38}$, it was also found that ion transport is dominated by the surface charge when the ionic concentration of the buffer solution is lower than $100 \mathrm{mM}$. However, little attention has been paid to the flow in these studies.

Jacobson et al. ${ }^{39}$ measured EOF velocity in nanochannels as small as $98 \mathrm{~nm}$ deep by using fluorescent dye (Rhodamine B), showing a $35 \%$ decrease of the electroosmotic mobility compared with that in microchannels. Hug ${ }^{40}$ developed a new method to fabricate nanofluidic chips based on silicon dioxide wafers and measured EOF velocity in both microchannels and nanochannels; however, the smallest channel size is just $200 \mathrm{~nm}$ which is not sufficient to study any unique transport phenomena in nanoscale, because there is essentially no overlap of EDLs in such a large nanochannel. Pennathur and Santiago 35 investigated experimentally electrokinetic transport phenomena in two nanochannels of $40 \mathrm{~nm}$ deep and $100 \mathrm{~nm}$ deep by using both the current monitoring method and the fluorescence imaging method. However, the width of these channels is about $1 \mu \mathrm{m}$, which is not in the nanoscale. These nanochannels were fabricated by conventional photolithography and dry etching method on quartz wafers. Haywood et al. ${ }^{41}$ measured EOF velocity in three glass nanochannels of $530 \mathrm{~nm}$ wide and $54 \mathrm{~nm}, 108 \mathrm{~nm}, 216 \mathrm{~nm}$ deep fabricated by combining photolithography, chemical etching, FIB (Focused lon Beam) lithography techniques and so on. Both Pennathur's and Haywood's results prove that EOF velocity in nanochannels decreases as EDLs get overlapped.

As reviewed above, even though several experimental studies have been conducted to investigate EOF in nanochannels, few of them worked on channels made by Polydimethylsiloxane (PDMS) which is the most widely used materials in microfluidic and nanofluidic research, furthermore, few of them worked with channel sizes smaller than $40 \mathrm{~nm}$ where significant EDL overlap may occur, especially in two dimensions. In the works reviewed above, very limited study of channel size effects on EOF velocity in nanochannels was presented. Therefore, the objective of this paper is to systematically investigate EOF in smaller single nanochannels made of PDMS in order to provide a better understanding of electrokinetic transport in PDMS nanochannels. In this work, a novel method is developed to fabricate single nanochannels on PDMS chips. EOF velocity in single PDMS nanochannnels with a dimension ranging from 20 to about $250 \mathrm{~nm}$ are measured systematically by the current slope method. The effects of channel size, concentration of electrolyte solution as well as the applied electric field on EOF velocity in nanochannels are studied. Zeta potential of the x-PDMS channel surfaces is also measured by using the current slope method. For comparison, a corresponding numerical study of EOF velocity in nanochannels is also presented in this paper.

\section{Theoretical analysis}

EOF in nanochannels with overlapped EDLs can be estimated by the following analytical solutions ${ }^{17}$ theoretically. Assuming that EOF transport between two sufficient large parallel plates separated by a distance of $2 d$, the electric potential distribution in the $y$ direction of the channel (perpendicular to the channel wall, and $y=0$ at the middle plane) with small degree of EDL overlapping can be written as ${ }^{17}$ :

$\psi(y)=\frac{4 k_{b} T}{z e}\left\{\tanh ^{-1}\left[\tanh \left(\frac{z e \zeta_{w}}{4 k_{b} T}\right) \exp \left(-k_{d}(y+d)\right)\right]\right\}+$
$\frac{4 k_{b} T}{z e}\left\{\tanh ^{-1}\left[\tanh \left(\frac{z e \zeta_{w}}{4 k_{b} T}\right) \exp \left(-k_{d}(d-y)\right)\right]\right\}$

where $k_{b}$ is the Boltzmann constant, $T$ is the temperature, $e$ is the unit charge, $z$ is the valence of the buffer solution $\left(z=z^{+}=\right.$ $-z^{-}=1$ for $\mathrm{KCl}$ ) and $k_{d}{ }^{-1}$ is the Debye length which is a function of the ionic concentration, $k_{d}$ is also referred as the Debye-Huckel parameter ${ }^{42}$.

$k_{d}{ }^{-1}=\lambda_{D}=\sqrt{\frac{\varepsilon_{0} \varepsilon_{r} k_{b} T}{2 n_{i \infty}(z e)^{2}}}$

Eq. 2

here $n_{i \infty}$ is the number density of ions in the bulk solution. $\varepsilon_{0}$ is the permittivity of vacuum and $\varepsilon_{r}$ is the dielectric constant of the electrolyte solution. Combining the Stokes equation under the condition of laminar flow, the EOF velocity distribution along the $y$ direction can be calculated by:

$\boldsymbol{v}_{\text {EOF }}(\boldsymbol{y})=-\frac{\varepsilon_{0} \varepsilon_{\mathrm{r}} \zeta_{w} \boldsymbol{E}_{e x}}{\mu}\left(1-\frac{\psi(y)}{\zeta_{w}}\right)$

Eq. 3

where $\boldsymbol{E}_{\boldsymbol{e} \boldsymbol{x}}$ is the externally applied electric field, $\zeta_{\boldsymbol{w}}$ is the zeta potential of the channel wall and $\mu$ is the viscosity of the electrolyte solution. In microchannels, the thickness of the EDL is negligible compared with the channel size, therefore, $\psi(y)$ becomes zero and Eq. 3 becomes the famous HelmholtzSmoluchowski equation ${ }^{42}$ :

$\boldsymbol{v}_{\text {EOF }}(\boldsymbol{y})=-\frac{\varepsilon_{0} \varepsilon_{\mathrm{r}} \zeta_{w}}{\mu} \boldsymbol{E}_{\boldsymbol{e x}}$

Eq. 4

Based on the profile of the EOF velocity, one can easily get the average velocity in the channel by integrating the velocity in the $y$ direction:

$\boldsymbol{v}_{\boldsymbol{a v}}=\frac{1}{2 d} \int_{-d}^{d} \boldsymbol{v}_{E O F}(y) d y$

Eq. 5

For very large degreees of EDL interactions or high zeta potential, Eq. 1 is not appliable anymore, and the electric potential along the $y$ direction of the channel can be calculated by ${ }^{17}$ :
$\psi(y)=\psi_{u}-\frac{2 k_{b} T}{z e} \ln \cos \left\{\cos ^{-1} \exp \left[\frac{z e}{2 k_{b} T}\left(\psi_{u}-\zeta_{w}\right)\right]-\right.$ $\left.\frac{k_{d}(y+d)}{2} \exp \left(\frac{z e}{2 k_{b} T} \psi_{u}\right)\right\}$
Eq. 6 
where $\psi_{u}$ is the electric potential at the middle plane, which can be calculated by applying the boundary condition: $y=0$, and the the following equation ${ }^{17}$ :

$\cos \left[\frac{k_{d}(y+d)}{2} \exp \left(\frac{z e}{2 k_{b} T} \psi_{u}\right)\right]=\exp \left[\frac{z e}{2 k_{b} T}\left(\psi_{u}-\zeta_{w}\right)\right] \quad$ Eq. 7

To study EOF in nanochannels numerically, direct numerical simulation by coupling Navier-Stokes equation, continuity equation and Poisson-Nernst-Plank equation are conducted to calculate the electric field, ionic concentration field as well as the flow field. The numerical simulation was conducted by using Comsol 4.3b (see the ESIt for the detail of the model). EOF velocity profiles and the average EOF velocities are also calculated and plotted according to the analytical solutions (Eq.1 to Eq.7) by using Matlab software to verify the reliability of the numerical model. In the numerical studies, ionic concentration from $10^{-4} \mathrm{M}$ to $0.5 \mathrm{M}$, channel height from 20 $\mathrm{nm}$ to $300 \mathrm{~nm}$, externally applied electric field ranging from 12.5 $\mathrm{V} / \mathrm{cm}$ to $50 \mathrm{~V} / \mathrm{cm}$ were tried. The zeta potential values were from the experimental results which will be discussed in the following parts.

\section{Experimental studies}

\section{Fabrication of single channel nanofluidic device}

In this work, single PDMS nanochannels were made by three steps. First of all, a single nanocrack is generated on a polystyrene surface by a solvent-induced cracking method ${ }^{43}$. The crack size can be controlled by adjusting the working parameters, such as the chemical reagent, heating temperature, heating time, volume of the reagent and so on. To make sure only one single nanocrack is generated, an artificial defect is marked on the polystyrene surface by using a microhardness testing system (LECO ${ }^{\circ}$, MHT series 200) before the solvent-induced cracking process is conducted. Secondly, the negative nanocrack pattern is transferred onto a piece of smooth cast 305 layer (Sculpture Supply Canada) by using the

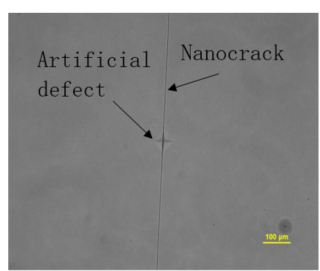

(A)

(C)

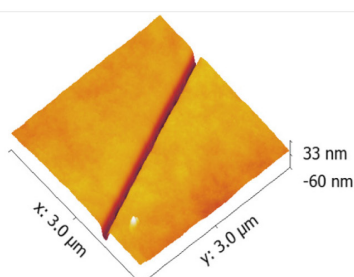

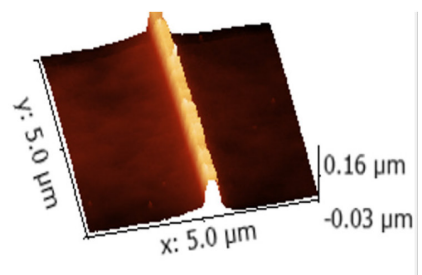

(B)

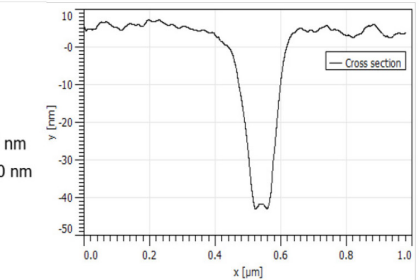

(D)
Fig. 1 Examples of nanocrack, nanochannel mold and PDMS nanochannel. (A) Single nanocrack generated on polystyrene surface; (B) Positive nanochannel mold on smooth cast surface; (C) Single nanochannel on PDMS; (D) Cross section of the PDMS nanochannel of $183 \pm 11 \mathrm{~nm}$ in width and
$42 \pm 6 \mathrm{~nm}$ in depth measured by AFM.

nanoimprint technique to form a positive nanochannel mold. Fig. 1(A) shows an example of single nanocrack generated on the polystyrene surface captured by optical microscope (Nikon, TE-2000). Fig. 1(B) is an example of a positive nanochannel mold on the smooth cast surface measured by AFM (Multimode ${ }^{\mathrm{TM}}$ SPM, Digital Instruments). Thirdly, the single nanochannel is replicated onto a $30 \mu \mathrm{m}$ thick extra hard PDMS (x-PDMS) ${ }^{44}$ layer followed by a 2 3 $\mathrm{mm}$ thick layer of regular PDMS (Sylgard 184). Finally, the bi-layer PDMS with the nanochannel is peeled off from the positive nanochannel mold. Fig. 1(C) shows an example of PDMS nanochannel replicated from the smooth cast nanochannel mold, and the cross section of this PDMS nanochannel was measured by AFM, as shown in Fig. 1 (D). Similar methodology to fabricate PDMS nanochannels can be found elsewhere ${ }^{45}$, in which the PDMS nanochannels are tuneable, however, channel arrays fabricated by this method

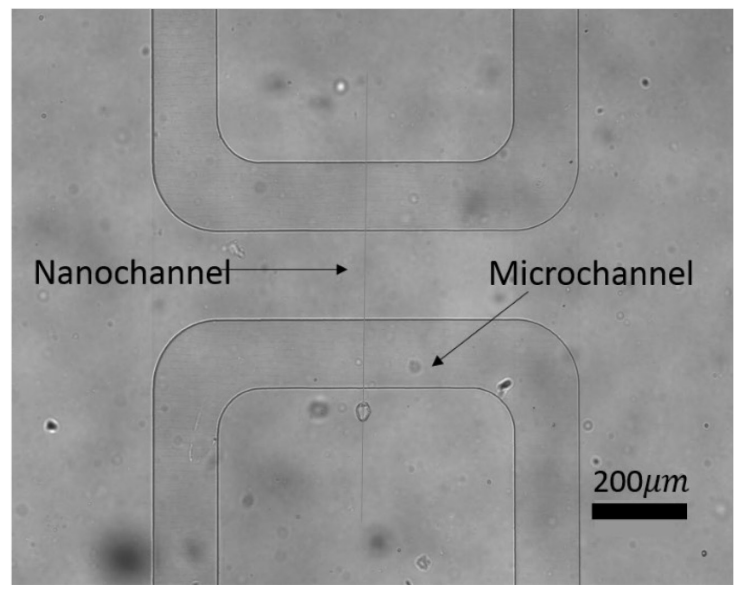

(A)

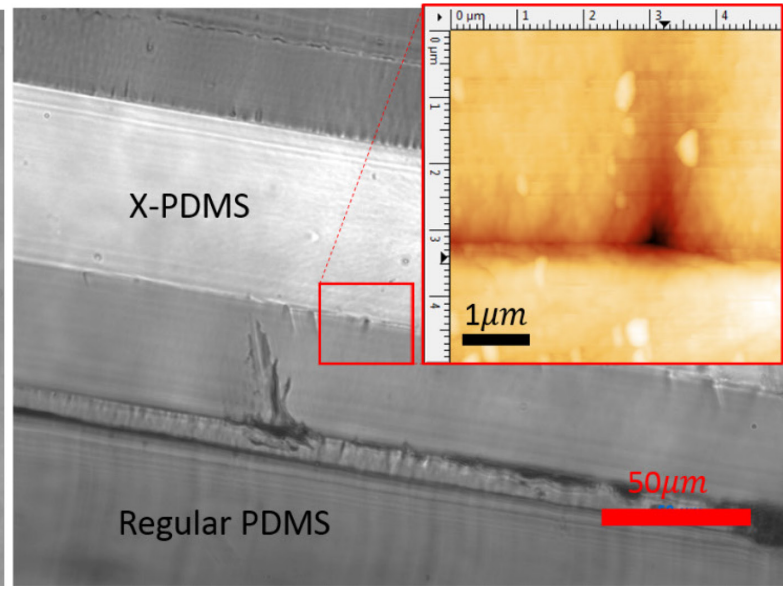

(B)

Fig. 2 (A) PDMS nanofluidic chip after bonding captured by the optical microscope; (B) Cross section of the nanochannel captured by the optical microscope and by the AFM. 
can hardly promise applications of single nanochannel systems in the electrokinetic transport studies. The working steps in the present paper make sure that only single nanochannels are developed.

In order to handle the liquid delivery to the single nanochannel, the nanochannel needs to be connected to a PDMS microchannel network. The SU8 photoresist microchannel molds are designed by AutoCAD ${ }^{\circledR}$ software and fabricated on silicon wafers by the standard photolithography technique; and PDMS microchannels are replicated from the SU8 channel mold by the soft lithography method. The microchannel system is composed of a pair of " $U$ " shape microchannels with a cross section of $150 \mu \mathrm{m}$ in width and $30 \mu \mathrm{m}$ in depth. The two " $U$ " shape microchannels are separated by 100 or $200 \mu m$. The nanochannels will bridge the gaps and form the final micro-nanofluidic chips, as shown in Fig. 2 (A). The PDMS nanochannel and the PDMS microchannel system can be bonded together by treating the bonding surfaces with plasma for $30 \mathrm{~s}$ (Harrick plasma ${ }^{\circledR}$, PDC-32G) and with the help of a homemade alignment system under the microscope. Fig. 2(A) is an example of micro-nanofluidic chip after bonding, and Fig. 2(B) shows a cross section of the nanochannel captured by the optical microscope and a zoomedin figure of this cross section measured by the AFM.

\section{EOF velocity measurement by the current slope method}

To measure EOF velocity in microchannels or in nanochannels, several methods have been developed, and these methods can be divided into two categories: imaging tracing method and current monitoring method. For the imaging tracing method, fluorescence dye or nanoparticles are diluted or mixed into electrolyte solutions to make the movements of the fluids visible. A camera or a photodetector will record the movements of the fluorescent dye or particles and calculate the flow velocity values. For example, Sadr et al. ${ }^{46}$ studied EOF in rectangular channels by using nanoparticles; Jacobson et al. ${ }^{39,41}$ measured EOF velocity through nanochannels by using Rhodamine dye. EOF in single nanochannels was also investigated by Pennathur and Santiago by imaging Rhodamine $B{ }^{35}$. Fluorescence imaging makes the velocity visible, however, diffusion of the fluorescent dye will result in a blurring boundary of the flow and an inaccuracy timing. Also, the fluorescent dye molecules and nanoparticles carry electrostatic charges, which make them subject to additional electrophoretic motion, and may affect the electric field in the nanochannel. These side effects must be considered and corrected in order to obtain the true EOF velocity by these methods.

The electrical current monitoring method can measure EOF velocity by monitoring the electric current change during replacement between two electrolyte solutions of slightly different conductivity. As a low conductivity solution replaces a high conductivity solution in a channel by the electroosmotic flow, the electric current decreases from an initial higher current value and eventually reaches a lower constant value when the high conductivity solution is completely replaced by the low conductivity solution in the channel. Ren et al 47 improved the current monitoring method by using the slope of the current change curve to minimize the inaccuracy involved in determining the starting time and the ending time of the current change. This improved method is also called the current slope method. The current slope method does not need the exact starting time and ending time of the solution displacement, and the average velocity can be calculated by the following equation:

$V_{a v}=\frac{k_{\text {slope }} \cdot L}{E_{e x} A\left(\lambda_{2}-\lambda_{1}\right)}$

Eq. 8

where $V_{a v}$ is the average velocity of the electroosmotic flow, $k_{\text {slope }}$ is the slope of the linear part, of the current change curve, $A$ is the cross section of the channel, $L$ is the length of the

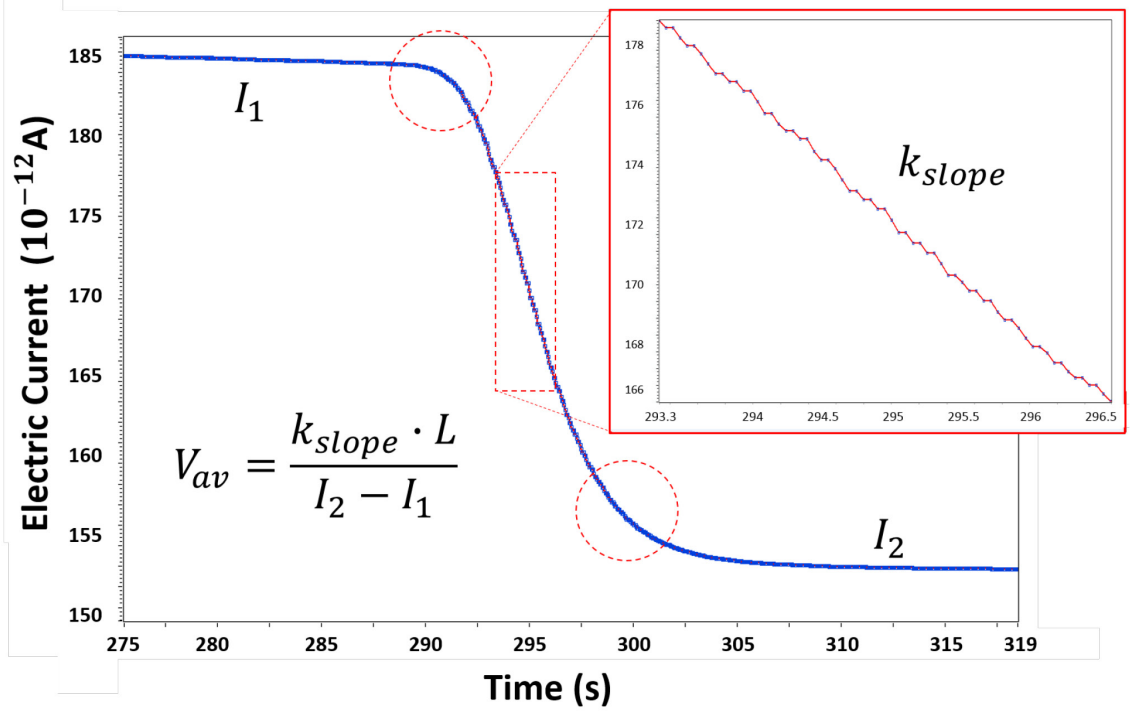

Fig. 3 An example of EOF velocity measurement in nanochannels by the current slope method. The current change is measured by replacing $100 \% 10^{-2} \mathrm{M} \mathrm{KCl}$ solution with $90 \% 10^{-2} \mathrm{M} \mathrm{KCl}$ solution with $25 \mathrm{~V} / \mathrm{cm}$ electric field applied in an $804 \mathrm{~nm}$ wide and $215 \mathrm{~nm}$ deep nanochannel. 


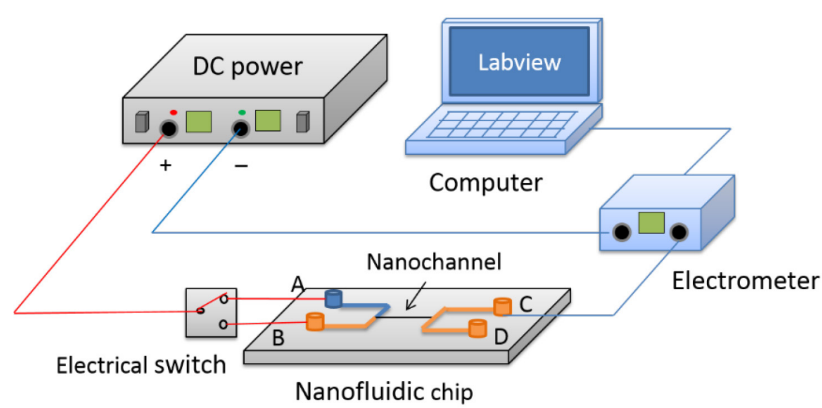

Fig. 4 Schematic of the current slope measurement system.

channel, $\lambda_{1}$ and $\lambda_{2}$ are the conductivity of the higher ionic concentration solution and lower ionic concentration solution, respectively.

In the single nanochannel chips used in this work, the length of the nanochannels is very short, and it is easy to measure the stable electric current values when the channel is filled with either the higher ionic concentration solution or the lower ionic concentration solution. As a consequence, Eq. (8) can be rewritten into the following form to calculate the EOF velocity in single nanochannels:

$V_{a v}=\frac{k_{\text {slope }} \cdot L}{I_{2}-I_{1}}$

Eq. 9

where $I_{1}$ and $I_{2}$ are the electric currents in the nanochannel filled with a higher ionic concentration solution and a slightly lower ionic concentration solution, respectively.

Fig. 3 shows an example of the current change curve measured by replacing $100 \% 10^{-2} \mathrm{M} \mathrm{KCl}$ solution with $90 \%$ $10^{-2} \mathrm{M} \mathrm{KCl}$ solution under $25 \mathrm{~V} / \mathrm{cm}$ electric field in an $804 \pm 13 \mathrm{~nm}$ wide and $215 \pm 7 \mathrm{~nm}$ deep nanochannel. At the beginning, only $100 \% 10^{-2} \mathrm{M} \mathrm{KCl}$ solution is flowing in the nanochannel, and the current is constant, $I_{1}$. After the $100 \%$ solution is replaced by the $90 \%$ concentration solution, the current decreases gradually and eventually reaches a constant lower current value, $I_{2}$, when the higher concentration solution is completely replaced by the lower concentration solution(see Fig. 3), due to the conductivity change. The most linear part of the current slope is magnified in the up-right corner of Fig. 3, which is used to represent the slope of the current change during entire displacement process. The nonlinear parts in the red circulars are caused by mixing and dispersion of the solutions at the starting and ending of the displacement.

Fig. 4 shows the layout of the current monitoring system. A DC power (Keithley, Model 6517A, Voltage module) is used to provide electric potential to the nanofluidic chip reservoirs through Platinum electrodes (Sigma-Aldrich ${ }^{\circ}$ ). Electrometer (Keithley, Model 6517A) connecting to the computer is used to record the current by Labview programs (National Instruments Corp.). To reduce the noise during the current measurement, a hand-made Faraday case is covered on the power switch and the nanofluidic chip.

For each set of measurement, a fresh nanofluidic chip was initially loaded with $\mathrm{KCl}$ solution of a given ionic concentration,
Tab. 1 Size of nanochannels used in the EOF velocity measurement. $\left(H_{D}\right.$ : Hydraulic diameter, A: cross area)

\begin{tabular}{|c|c|c|c|c|}
\hline $\begin{array}{c}\text { Channel } \\
\text { No. }\end{array}$ & Depth (nm) & Width (nm) & $\mathrm{H}_{\mathrm{D}}(\mathrm{nm})$ & $A\left(n m^{2}\right)$ \\
\hline 1 & $241 \pm 11$ & $867 \pm 20$ & 225 & 104570 \\
\hline 2 & $215 \pm 7$ & $804 \pm 13$ & 201 & 86811 \\
\hline 3 & $183 \pm 12$ & $798 \pm 19$ & 174 & 73115 \\
\hline 4 & $149 \pm 15$ & $795 \pm 18$ & 144 & 59315 \\
\hline 5 & $130 \pm 16$ & $714 \pm 19$ & 126 & 46630 \\
\hline 6 & $114 \pm 17$ & $716 \pm 21$ & 111 & 40907 \\
\hline 7 & $104 \pm 9$ & $757 \pm 25$ & 101.5 & 39417 \\
\hline 8 & $94 \pm 11$ & $609 \pm 9$ & 92 & 28343 \\
\hline 9 & $89 \pm 2$ & $626 \pm 17$ & 87 & 27857 \\
\hline 10 & $81 \pm 5$ & $532 \pm 9$ & 79 & 21546 \\
\hline 11 & $67 \pm 7$ & $640 \pm 22$ & 66 & 21440 \\
\hline 12 & $58 \pm 2$ & $404 \pm 15$ & 57 & 11716 \\
\hline 13 & $50 \pm 2.5$ & $232 \pm 11$ & 39 & 5800 \\
\hline 14 & $42 \pm 5.6$ & $183 \pm 11$ & 40 & 3843 \\
\hline 15 & $32 \pm 4$ & $106 \pm 9$ & 29 & 1696 \\
\hline 16 & $20 \pm 5$ & $63 \pm 10$ & 18.3 & 630 \\
\hline
\end{tabular}

ranging from $10^{-4} \mathrm{M}$ to $0.5 \mathrm{M}$ in the experiments. The electrolyte solutions were prepared by dissolving $99.9 \% \mathrm{KCl}$ (Fisher Scientific ${ }^{\circ}$ ) in pure water (Mini Q, Direct-Q 3) with resistivity of $18.2 \mathrm{M} \Omega \cdot \mathrm{cm}$ at $25^{\circ} \mathrm{C}$. Then, DC power was applied on the nanochannel through reservoir $A$ and $C$ (see Fig. 4), and the current was recorded by the computer simultaneously. After the current became constant, the solution in reservoir $B$ was replaced by another kind of $\mathrm{KCl}$ solution with a slightly lower concentration with electric field applied through reservoirs B and C. Under the applied electrical field, the solution from reservoir $B$ would be pumped into the microchannel and then into the nanochannel because of the electroosmotic flow. As a consequence, the solution with a higher concentration in the nanochannel would be replaced by the lower concentration solution gradually by EOF, and the electric current would decrease gradually until reaching another plateau. The length of the microchannel involved in the nanofluidic chip is about $1.5 \mathrm{~cm}$ in total, and the nanochannel length ranges from 100 to $200 \mu \mathrm{m}$. It can be easily evaluated that the voltage drop in the microchannels is only about $0.5 \%$ of the total voltage applied, which is negligible. Moreover, ion concentration polarization may occur at the micro-nanochannel interfaces. In this work, the electric field applied in the experiments was lower than $50 \mathrm{~V} / \mathrm{cm}$ and the ion concentration polarization is very weak. In addition, the period of time of the electrolyte displacement was only few seconds which is much shorter than that of the propagation process of the ion concentration polarization. As a consequence, in this work the ion concentration polarization effects is assumed to be negligible. Similar assumptions has been applied and proved by Haywood et al ${ }^{41}$. 
It should be noted that the electric current value in nanochannels decreases with the decrease of the ionic concentration of the solutions, which makes the current measurement harder for low concentrations solutions. In this paper, $5 \%$ concentration difference was applied for both $0.5 \mathrm{M}$ and $0.1 \mathrm{M}$ concentration cases; $10 \%$ concentration difference was used for $0.01 \mathrm{M}$ and $10^{-3} \mathrm{M}$ cases; and $20 \%$ concentration difference was utilized in the case of $10^{-4} \mathrm{M}$ solution during the displacements. For each working condition, i.e. one given solution, one given electric field and one given nanochannel size, the current slope measurement was repeated for at least 5 times in 5 independent new chips. EOF velocity was calculated by using Eq. 9 .

In this study, 16 nanochannels with different sizes, width ranging from approximately $60 \mathrm{~nm}$ to $860 \mathrm{~nm}$, and depth ranging from $20 \mathrm{~nm}$ to $241 \mathrm{~nm}$, were tested. Tab. 1 gives the geometric parameters of these nanochannels, including the width, depth, hydraulic diameter and the cross-section area values. The length of the nanochannels connecting the two " $U$ " shaped microcahnnels in the nanofluidic chips is $200 \mu \mathrm{m}$ for channel 1 to channel 12 , and $100 \mu m$ for channel 13 to channel 16. All the PDMS nanochannels were measured by AFM for at least five times to characterize the channel size. A microchannel of $5 \mu \mathrm{m}$ deep, $50 \mu \mathrm{m}$ wide and $1 \mathrm{~cm}$ long fabricated by traditional photolithography method was also used to study the EOF velocity in microchannels and to measure the zeta potentials of $x$-PDMS channel surfaces under different ionic concentrations. All the experiments were conducted in room temperature of about $25^{\circ} \mathrm{C}$.

\section{Results and discussion}

\section{Zeta potential measurement in x-PDMS microchannels}

Zeta potential is used to predict EOF velocity in microchannels by Helmholtz-Smoluchowski equation. As a comparison work, EOF velocity of $0.5 \mathrm{M}, 0.1 \mathrm{M}, 10^{-2} \mathrm{M}, 10^{-3} \mathrm{M}$ and $10^{-4} \mathrm{M} \mathrm{KCl}$ solution in the microchannel of $5 \mu \mathrm{m}$ deep, $50 \mu \mathrm{m}$ wide and $1 \mathrm{~cm}$ long was also measured by the current slope method. The zeta potentials of the channel surfaces at each concentrations are calculated by using the Helmholtz-Smoluchowski equation (Eq. 4) and the EOF velocity values. During the calculation, the permittivity and viscosity of the solutions are treated as constants (see ESI), and the zeta potential of the $x$-PDMS microchannel surfaces is fitted as a function of the ionic concentration (as shown in Fig. 5):

$y[m V]=-8.27448+2.13367 * \ln \left(c[M]-3.42286 * 10^{-5}\right)$

Eq. 10

Zeta potential of the $x$-PDMS channel surfaces under the condition of any ionic concentration between $10^{-4} \mathrm{M}$ and $0.5 \mathrm{M}$ in the simulation part of this study was calculated by using Eq. 10. Here, the assumption is that the zeta potential is dominated by the concentration and does not change with the overlapping of EDLs. To be noted, overlapping of EDLs affects zeta potential of nanochannel surfaces, and a more accurate model has been

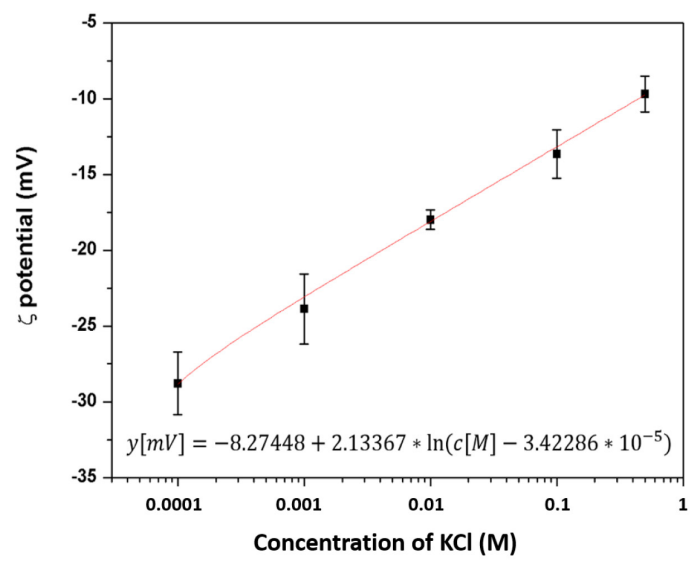

Fig. 5 Zeta potential of $x$-PDMS microchannels of $5 \mu \mathrm{m}$ deep, $50 \mu \mathrm{m}$ wide and $1 \mathrm{~cm}$ long with different $\mathrm{KCl}$ solutions ranging from $10^{-4} \mathrm{M}$ to $0.5 \mathrm{M}$ is calculated by Helmholtz-Smoluchowski equation.

developed by Ma et al. ${ }^{27}$ The model shows that the deviation of zeta potential for the cases of high degree EDL interactions under the condition of low $\mathrm{pH}$ values (for example $\mathrm{pH}=4$ ) is significant ${ }^{27}$. However, in our cases, the $\mathrm{pH}$ values are higher, about 5.7, and the interactions between EDLs are much weaker compared with that of the model with large deviation of zeta potential, which indicates that the percentage deviation of the zeta potential in our work is very small and negligible.

\section{Channel size effects}

Single PDMS nanochannels with depth ranging from $20 \mathrm{~nm}$ to $241 \mathrm{~nm}$ as well as a microchannel of a cross-section $50 \times 5 \mu \mathrm{m}$ were used to study the channel size effects upon the EOF velocity in nanochannels. During the experiments, all the channels initially were filled with $100 \% 10^{-2} \mathrm{M} \mathrm{KCl}$ solution and $100 \% 10^{-3} \mathrm{M} \mathrm{KCl}$ solution; and then the initial solutions were replaced by $90 \% 10^{-2} \mathrm{M}$ and $90 \% 10^{-3} \mathrm{M} \mathrm{KCl}$ solutions under 25 $\mathrm{V} / \mathrm{cm}$ electric field. Fig. 6 shows the experimental results and the numerical simulation results. To simplify the figures, only the smallest dimension (the depth) of the channels is plotted as the $x$-axis in the figures, the width of the channels is labelled above or below the error bars (see Fig. 6(A)). Numerical simulation results are also plotted on Fig. $6(A)$, in which the red dashed line depicts the simulation results of the $10^{-3} \mathrm{M}$ cases and the black dashed line is the simulation results of the $10^{-2} \mathrm{M}$ cases. From Fig. $6(\mathrm{~A})$, it is obvious that, in relatively large nanochannels, i.e., when the depth (the smallest dimension) is larger than $100 \mathrm{~nm}$ for the case of $10^{-3} \mathrm{M}$ solution or larger than $50 \mathrm{~nm}$ for the case of $10^{-2} \mathrm{M}$ solution, the EOF velocity values are independent of the channel size, about $40 \mu \mathrm{m} / \mathrm{s}$ and approximate $30 \mu \mathrm{m} / \mathrm{s}$, respectively. However, when the channel size becomes smaller, i.e., when the depth (the smallest dimension) is smaller than $100 \mathrm{~nm}$ for the case of $10^{-3} \mathrm{M}$ solution or smaller than $50 \mathrm{~nm}$ for the case of $10^{-2} \mathrm{M}$ solution, EOF velocity in the nanochannels for both concentration values becomes size dependent and decreases with the decrease of the channel size. The experimental results in Fig. 6(A) agree with the numerical simulation results well. The profiles of the EOF velocity in the nanochannels for both $10^{-2} \mathrm{M}$ cases and $10^{-3} \mathrm{M}$ 


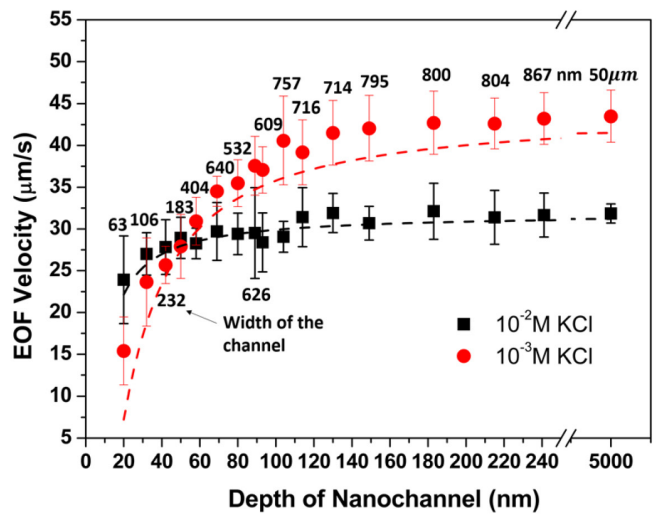

(A)

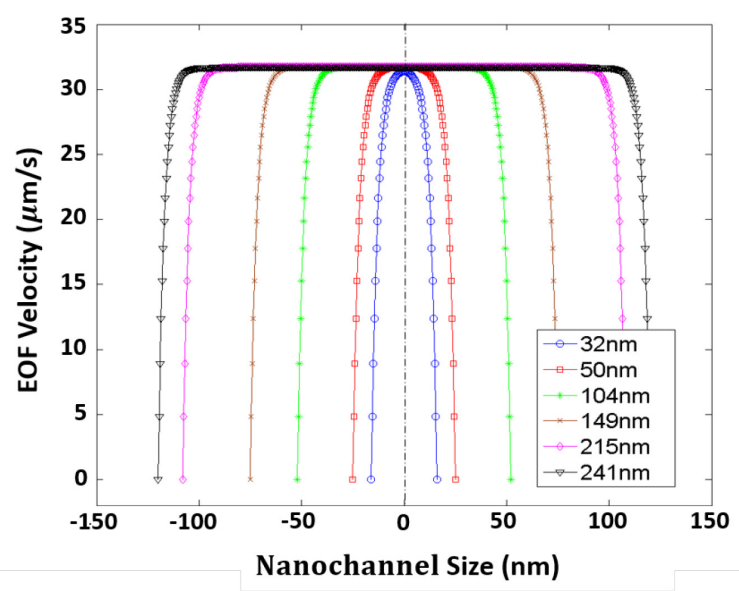

(B)

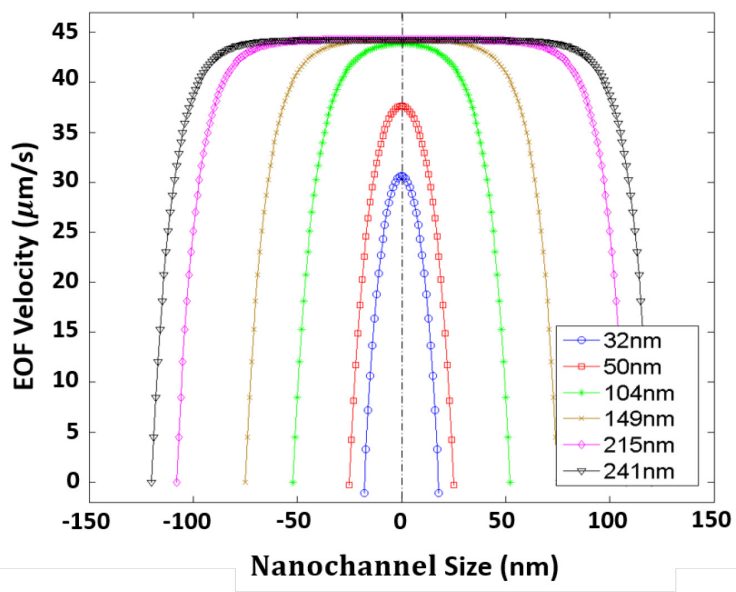

(C)

Fig. 6 (A) Experimental results and numerical simulation results of channe size effects on EOF velocity in nanochannels. In the experiments, EOF velocity of $10^{-2} \mathrm{M} \mathrm{KCl}$ and $10^{-3} \mathrm{M} \mathrm{KCl}$ solutions in 16 nanochannels with depth ranging from $20 \mathrm{~nm}$ to $241 \mathrm{~nm}$ and a microchannel with a crosssection of $50 \times 5 \mu \mathrm{m}$ are measured under the electric field of $25 \mathrm{~V} / \mathrm{cm}$. The width of the channels are labelled near the error bars of each data point The red dashed line depicts the simulation results of $10^{-3} \mathrm{M}$ cases and the profiles in nanochannels with $10^{-2} \mathrm{M} \mathrm{KCl}$ solution; (C) EOF velocity profiles in nanochannels with $10^{-3} \mathrm{M} \mathrm{KCl}$ solution. cases are plotted in Fig. 6(B) and Fig. 6(C) by the numerical method.

For the $10^{-2} \mathrm{M}$ cases, EOF velocity in all the larger nanochannels is essentially constant, about $30 \mu \mathrm{m} / \mathrm{s}$, which is identical with the velocity in the $50 \times 5 \mu \mathrm{m}$ microchannel (see Fig. 6(A)). Channel size has little effect on the EOF velocity, because the EDL is very thin in comparison with the relatively large nanochannel size. For $10^{-2} \mathrm{M} \mathrm{KCl}$ solution, the Debye length $\lambda_{D}\left(1 / k_{d}\right)$ is only about $3 \mathrm{~nm}$, and the thickness of the diffuse layer of electric double layer is usually 3 to 5 times of $\lambda_{D}$ $42,10 \sim 15 \mathrm{~nm}$, which means no overlap of EDLs occurs in nanochannel larger than $30 \mathrm{~nm}$. Therefore, the EOF velocity in these nanochannels is just as large as that in the microchannels, and Helmholtz-Smoluchowski equation is still applicable. However, in reality, the average EOF velocity still shows a slightly decreasing trend when the channels size becomes smaller than, for example, $50 \mathrm{~nm}$, as showing in Fig. 6(A). This is because the percentage of the nanochannel cross-section occupied by the EDL increases when the channel size becomes smaller. When the channel size is smaller than $30 \mathrm{~nm}$, for example, in the $20 \mathrm{~nm}$ deep nanochannel, a weak degree of overlap of EDLs occurs, which will further reduce the EOF velocity to about $24 \mu \mathrm{m} / \mathrm{s}$.

Similarly, for the $10^{-3} \mathrm{M}$ cases, $\lambda_{D}$ is about $10 \mathrm{~nm}$ and the thickness of the diffuse layer of electric double layer is about 30 $\sim 50 \mathrm{~nm}$. Consequently, EDLs are likely to get overlapped in the nanochannels with size smaller than $100 \mathrm{~nm}$ and the EOF velocity will decrease accordingly. As expected, from Fig. 6(A) one can see that EOF velocity in nanochannels with $10^{-3} \mathrm{M}$ solution decreases gradually as the channels size becomes smaller than $100 \mathrm{~nm}$. The velocity profiles in Fig. 6(C) also show that the maximum velocity value in the $104 \mathrm{~nm}$ deep nanochannel is slightly smaller than that in the microchannel as shown in Fig. 6(A).

In addition, in most cases of this study, the nanochannel size in the width direction is much larger than that in the depth direction, and therefore, it is impossible for the EDLs to get overlapped in the channel width direction; consequently, the channel width has little effect on the EOF velocity. However, for the smallest nanochannel studied in this work, the channel is 20 $\mathrm{nm}$ deep and $63 \mathrm{~nm}$ wide. In this case, EDLs can get overlapped weakly in the width direction in addition to the depth direction. As shown in Fig. 6(A), the EOF velocity is approximate $15 \mu \mathrm{m} / \mathrm{s}$, only about one third of that in the microchannel.

\section{Ionic concentration effects}

Fig. 7 shows the experimental results (solid points with error bars) and numerical simulation results (dashed lines) of ionic concentration effects on the EOF velocity in 6 nanochannels and one microchannel. $\mathrm{KCl}$ solution with concentrations ranging from $0.5 \mathrm{M}$ to $10^{-4} \mathrm{M}$ were studied in these channels. The applied electric field for all the cases is $25 \mathrm{~V} / \mathrm{cm}$. Apparently, EOF velocities of the $0.5 \mathrm{M}$ solution in all the nanochannels and the microchannel are essentially the same, about $17 \mu \mathrm{m} / \mathrm{s}$, because, at this high ionic concentration, the thickness of the EDL is extreme thin $\left(\lambda_{D} \approx 0.43 \mathrm{~nm}\right)$ and there is no overlap of EDLs in all the channels. As the ionic concentration decreases to 


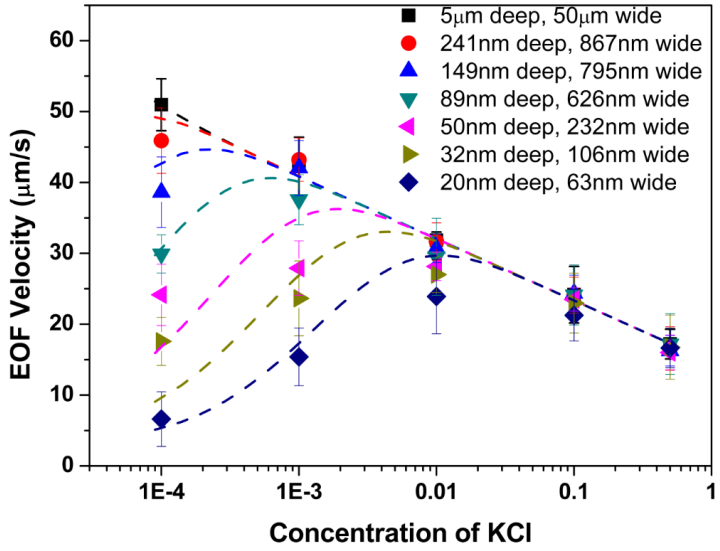

Fig. 7 Concentration effects on the EOF velocity. EOF velocity of $10^{-4} \mathrm{M}, 10^{-3}$ $\mathrm{M}, 0.01 \mathrm{M}, 0.1 \mathrm{M}$ and $0.5 \mathrm{M} \mathrm{KCl}$ solution in five nanochannels $(241 \times 867 \mathrm{~nm}$ $149 \times 795 \mathrm{~nm}, 89 \times 626 \mathrm{~nm}, 50 \times 232 \mathrm{~nm}$ and $32 \times 106 \mathrm{~nm})$ and microchannel $(5 \times 50 \mu \mathrm{m})$ were measured systematically. The applied the experimental results and the dashed lines illustrates the corresponding simulation results.

$0.1 \mathrm{M}$ and $10^{-2} \mathrm{M}$, EOF velocity in the channels increases with the concentration inversely due to the increasing zeta potential as shown in Fig. 5. However, a lower ionic concentration will also contribute to a thicker EDL, which will in turn give rise to overlapped EDLs in smaller nanochannels and consequently a smaller EOF velocity. However, when the ionic concentration becomes further lower to $10^{-3} \mathrm{M}$ and $10^{-4} \mathrm{M}$, channels of different sizes behavior differently in terms of EOF velocity. For example, in the microchannel, EOF velocity keeps increasing from about $42 \mu \mathrm{m} / \mathrm{s}$ to almost $53 \mu \mathrm{m} / \mathrm{s}$ while the ionic concentration decreases from $10^{-3} \mathrm{M}$ to $10^{-4} \mathrm{M}$, following the trend of the zeta potential in Fig. 5. For the $241 \mathrm{~nm}$ deep and $149 \mathrm{~nm}$ deep nanochannels, the velocity still increases with the concentration inversely for the $10^{-3} \mathrm{M}$ case, but decreases slightly for the $10^{-4} \mathrm{M}$ case due to weak overlap of EDLs. For nanochannels smaller than $89 \mathrm{~nm}$, overlapped EDLs will dominate the EOF transport, and the EOF velocity decreases dramatically even though the ionic concentration decreases. For example, in the $20 \mathrm{~nm}$ deep and $63 \mathrm{~nm}$ wide nanochannel with $10^{-4} \mathrm{M}$ solution, $\lambda_{D} \approx 30 \mathrm{~nm}$, EDLs are overlapped in both the depth direction and the width direction; consequently, EOF velocity is reduced to only about $1 / 9$ of that in the microchannel under the same applied electric field. It is obvious that the experimental results and the numerical simulation results in Fig. 7 show the similar trends and indicate that the theoretical model of EOF with the overlapped EDLs works well. From both figures one can conclude that, in relatively larger nanochannels without overlapped EDLs, the EOF velocity depends on the ionic concentration; in smaller nanochannels with overlapped EDLs, the EOF velocity depends on both the ionic concentration and the channel size.

From the results one can see that EOF velocity in PDMS nanochannels depends highly on the ionic concentration. Similar properties of EOF in glass nanochannels have also been proved experimentally by Pennathur ${ }^{35}$ and Haywood et al ${ }^{41}$, and the electroosmotic mobility values in their works are higher than that in the present cases under the conditions of same concentration values and channel sizes due to the surface zeta potential difference between glass and X-PDMS material. However, all the results follow the EDL overlapping theory, as a result, one can also predict EOF transport in nanochannels, nanomembranes, nanoporous made of other materials such as silicon, polycarbonate and so on accordingly, which is essential to the applications of nanofluidics.

\section{Electric field effects}

Fig. 8 shows the electric field effects on the EOF velocity in nanochannels. In the experiments, EOF velocities of $10^{-2} \mathrm{M} \mathrm{KCl}$ solution and $10^{-3} \mathrm{M} \mathrm{KCl}$ solution in 5 nanochannels as well as in one microchannel with applied electric fields ranging from 12.5 $\mathrm{V} / \mathrm{cm}$ to $50 \mathrm{~V} / \mathrm{cm}$ were studied. As showing in both Fig. 8(A) and Fig. 8(B), it is obvious that EOF velocity in both the microchannel and the nanochannels increases linearly with the applied electric field. The slope of the velocity vs electric field curve is the electroosmotic mobility. From Fig. 8(A), one can see that the electroosmotic mobility decreases by a little with the

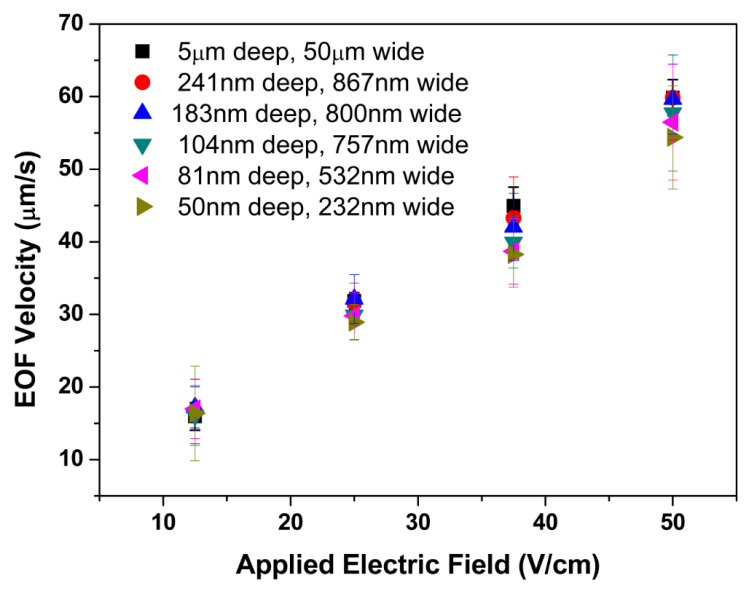

(A)

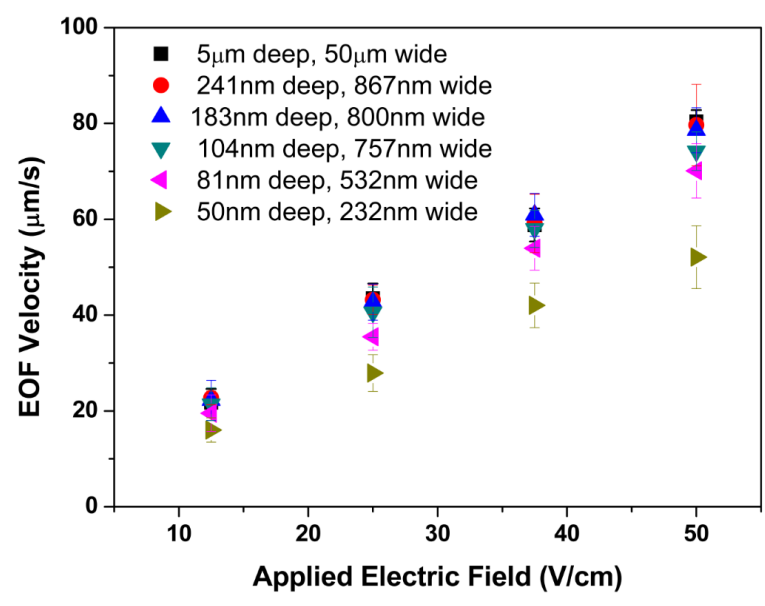

(B)

Fig. 8 Electric field effects on the EOF velocity. In the experiments, EOF velocity of (A) $10^{-2} \mathrm{M} \mathrm{KCl}$ solution and (B) $10^{-3} \mathrm{M} \mathrm{KCl}$ solution in 5 nanochannels and one microchannel are measured. The applied electric fields are $12.5 \mathrm{~V} / \mathrm{cm}, 25 \mathrm{~V} / \mathrm{cm}, 37.5 \mathrm{~V} / \mathrm{cm}$ and $50 \mathrm{~V} / \mathrm{cm}$ respectively. 
decreasing channel size even though no overlap of EDLs exists. As discussed above, the slight decrease of the electroosmotic mobility in the small nanochannels is due to the increase of the percentage of the channel cross-section occupied by EDLs when the channel size becomes smaller. For $10^{-3} \mathrm{M} \mathrm{KCl}$ solution, no overlap of EDLs occurs in nanochannels larger than $100 \mathrm{~nm}$, as shown in Fig. 8(B), EOF velocity in the nanochannels larger than $104 \mathrm{~nm}$ is essentially the same as that in the microchannel under the same applied electric field. However, Fig. 8 (B) shows that the electroosmotic mobility decreases when the channel size becomes smaller than $104 \mathrm{~nm}$, because EDLs get overlapped. This is evident for the case of $50 \mathrm{~nm}$ deep nanochannels.

\section{Conclusions}

This paper presents a systematic study of electroosmotic flow (EOF) for the first time in single PDMS nanochannels with a dimension as small as $20 \mathrm{~nm}$ by using the current slope method. 16 nanochannels with different sizes as well as a microchannel were tested in the experiments. Zeta potentials of the $x$-PDMS microchannel surfaces in $\mathrm{KCl}$ solutions with different concentrations were also measured by the current slope method. The results show that EOF velocity in nanochannels increases with the applied electric filed linearly, and EOF velocity in nanochannels with overlapped EDLs is smaller than that in microchannels under the same applied electric field. EOF velocity in relatively large nanochannels without overlapped EDLs is independent of channel size and can be calculated by Helmholtz-Smoluchowski equation. However, EOF velocity in smaller nanochannels with overlapped EDLs is strongly dependent on both the nanochannel size and the ionic concentration of the electrolyte solution. The experimental results and numerical simulation results presented in this paper provide improved understanding of electrokinetic transport through nanochannels.

\section{Acknowledgements}

The authors wish to thank the financial support of the Natural Sciences and Engineering Research Council (NSERC) of Canada through a research grant to $\mathrm{Dr}$. Li.

\section{References}

Y. Ai, M. Zhang, S. W. Joo, M. a. Cheney and S. Qian, J. Phys. Chem. C, 2010, 114, 3883-3890. L. J. Cheng and L. J. Guo, ACS Nano, 2009, 3, 575-584. P. Y. Apel, I. V Blonskaya, O. L. Orelovitch, P. Ramirez and B. a Sartowska, Nanotechnology, 2011, 22, 175302.

4 H. S. White and A. Bund, Langmuir, 2008, 24, 2212-2218.

5 Q. Pu, J. Yun, H. Temkin and S. Liu, Nano Lett., 2004, 4, 1099-1103.

6 X. Xuan and D. Li, Electrophoresis, 2007, 28, 627-34.

7 F. H. J. Van Der Heyden, D. Stein and C. Dekker, Phys. Rev.
Lett., 2005, 95, 9-12.
8

9

10

11

12

13

14

15

16

17

18

19

20

21

22

23

24

25

26

27

28

29

30

31

32

33

34

35

36
M. B. Andersen, H. Bruus, J. P. Bardhan and S. Pennathur, J. Colloid Interface Sci., 2011, 360, 262-71.

H. Daiguji, Chem. Soc. Rev., 2010, 39, 901-911.

A. Piruska, M. Gong, J. V Sweedler and P. W. Bohn, Chem. Soc. Rev., 2010, 39, 1060-72.

W. Sparreboom, a van den Berg and J. C. T. Eijkel, Nat. Nanotechnol., 2009, 4, 713-720.

Z. Yuan, A. L. Garcia, G. P. Lopez and D. N. Petsev, Electrophoresis, 2007, 28, 595-610.

S. Movahed and D. Li, Electrophoresis, 2011, 32, 12591267.

W. Qu and D. Li, J. Colloid Interface Sci., 2000, 224, 397407.

D. Burgreen and F. R. Nakache, J. Phys. Chem., 1964, 68, 1084-1091.

C. L. Rice and R. Whitehead, J. Phys. Chem., 1965, 69, 4017-4024.

R. J. Hunter, Zeta potential in colloid science: principles and applications, Academic press, 1981.

S. Bhattacharyya, Z. Zheng and a. T. Conlisk, J. Fluid Mech., 2005, 540, 247.

F. Baldessari, J. Colloid Interface Sci., 2008, 325, 526-538.

S. Melchionna and U. Marini Bettolo Marconi, EPL

(Europhysics Lett., 2011, 95, 44002.

E. González-Tovar, M. Lozada-Cassou and W. Olivares, J. Chem. Phys., 1991, 94, 2219.

Z. Zheng, D. J. Hansford and A. T. Conlisk, Electrophoresis, 2003, 24, 3006-3017.

S. Pennathur and J. Santiago, Anal. Chem., 2005, 77, 67726781.

R. Qiao and N. R. Aluru, Langmuir, 2005, 21, 8972-8977.

D. Hildreth, J. Phys. Chem., 1970, 74, 2006-2015.

Y. S. Choi and S. J. Kim, J. Colloid Interface Sci., 2009, 333, 672-678.

Y. Ma, L. H. Yeh, C. Y. Lin, L. Mei and S. Qian, Anal. Chem., 2015, 87, 4508-4514.

J. Liu, M. Wang, S. Chen and M. O. Robbins, J. Comput. Phys., 2010, 229, 7834-7847.

F. Sofos, T. E. Karakasidis and A. Liakopoulos, Int. J. Heat Mass Transf., 2010, 53, 3839-3846.

S. Levine, J. R. Marriott, G. Neale and N. Epstein, J. Colloid Interface Sci., 1975, 52, 136-149.

Y. Chen, Z. Ni, G. Wang, D. Xu and D. Li, Nano Lett., 2008, 8, 42-8.

L.-H. Yeh, S. Xue, S. W. Joo, S. Qian and J.-P. Hsu, J. Phys. Chem. C, 2012, 116, 4209-4216.

R. Qiao and N. R. Aluru, J. Chem. Phys., 2003, 118, 4692.

F. Baldessari, J. Colloid Interface Sci., 2008, 325, 539-546.

S. Pennathur and J. G. Santiago, Anal. Chem., 2005, 77, 6782-6789.

A. N. Chatterjee, D. M. Cannon, E. N. Gatimu, J. V. Sweedler, N. R. Aluru and P. W. Bohn, J. Nanoparticle Res., 2005, 7, 507-516.

D. Stein, M. Kruithof and C. Dekker, Phys. Rev. Lett., 2004, 93, 035901.

C. Duan and A. Majumdar, Nat. Nanotechnol., 2010, 5, 
848-852.

S. C. Jacobson, J. P. Alarie and J. M. Ramsey, Micro Total Anal. Syst., 2001, 57-59.

40 T. S. Hug, N. F. de Rooij and U. Staufer, Microfluid. Nanofluidics, 2006, 2, 117-124.

41 D. G. Haywood, Z. D. Harms and S. C. Jacobson, Anal. Chem., 2014, 86, 11174. D. Li, Electrokinetics in microfluidics, Academic Press, 2004, vol. 2.

43 R. Peng and D. Li, Biomicrofluidics, 2015, 9, 024117.

44 M. Verschuuren, 2009.

45 D. Huh, K. L. Mills, X. Zhu, M. A. Burns, M. D. Thouless and S. Takayama, Nat. Mater., 2007, 6, 424-428. R. Sadr, M. Yoda, Z. Zheng and A. T. Conlisk, J. Fluid Mech., 2004, 506, 357-367.

L. Ren, C. Escobedo-Canseco and D. Li, J. Colloid Interface Sci., 2002, 250, 238-42. 